\title{
Análisis de la construcción de la identidad terapéutica " alternativa" en el contexto del campo de la salud en Buenos Aires
}

\author{
MARIANA BORDES \\ Doctoranda en Ciencias Sociales (Universidad de Buenos Aires) \\ Miembro Investigador del Centro Argentino de Etnología Americana - CONICET \\ y del Instituto de Investigaciones Gino Germani (UBA) \\ Profesora de la UBA \\ Buenos Aires, Argentina \\ marianabordes@yahoo.com.ar
}

\begin{abstract}
Resumen
Este artículo presenta algunos elementos conceptuales para el análisis de las terapias alternativas, habitualmente asociadas al movimiento $\mathrm{N}$ ew A ge, tomando como conceptos centrales la idea de campo de la salud y la construcción de identidades colectivas. R especto a este último punto, el texto se centra en la teoría de la hegemonía elaborada por Ernesto Laclau, lo que permite indagar cómo la identidad alternativa se construye en términos distintivos a partir de operaciones de equivalenciación, reconversión o exclusión de determinados significantes que pertenecen a las formaciones discursivas de otros enfoques médicos. Se llega a la conclusión de que las características propias de esta propuesta identitaria habilitan - en virtud de su carácter flexible - al desarrollo de estrategias distintivas de legitimación por parte de los especialistas, lo que permite comprender la heterogeneidad identitaria que atraviesa a este universo simbólico. A I mismo tiempo, esa 'flexibilidad' ofrece el terreno analítico para discutir acerca de las potencialidades de subversión (o, por el contrario, de subsunción) respecto a la lógica dominante impuesta, en el campo de la salud, desde la biomedicina o medicina científica.
\end{abstract}

Palabras claves: terapias alternativas; identidades colectivas; Laclau; Buenos A ires.

\section{Introducción}

D ESDE HACE YA ALGUNAS DÉCADAS, principalmente a partir de las profundas tran formaciones estructurales que impactaron a escala mundial desde la década de 1970, el ámbito de las ciencias sociales se ha centrado en la tarea de redefinir en términos críticos las concepciones esencial istas referidas a los conceptos de sujeto e identidad.

En ese sentido, la crisis que atravesaron las concepciones que representaban a los grandes sujetos sociales, así como la emergencia de una pluralidad de 'nuevas' identidades étnicas, políticas, religiosas, se tradujo en la introducción de nuevas propuestas analíticas, sobre todo vinculadas al psicoanálisis, el postestructuralismo y la semiótica.

Ese giro epistemológico que se opera en el marco de las ciencias sociales es de especial relevancia en tanto las identidades, al dejar de ser consideradas como totalidades suturadas cuya especificidad es predefinida de antemano, pasan a inscribirse en un plano de indagación propiamente político, en la medida en que: 1) son consideradas en su carácter siempre incompleto, abierto y conflictivo, susceptible de ser 
transformadas en el marco de la articulación siempre contingente de nuevas relaciones; y 2 ) instituyen en el centro del análisis el carácter constitutivo del 0 tro - concebido como diferencia - en la definición de toda identidad.

Pues bien, cabe destacar que el espacio social relativo a los procesos de salud-enfermedad-atención no escapa a esta transformación de las viejas certezas identitarias verificada en diferentes ámbitos de estudio. Como indica de modo sugerente Bourdieu (2004), si pensamos la atención de la salud en términos de campo, resulta ineludible constatar un proceso de reconfiguración estructural en el que la emergencia de opciones terapéuticas novedo sas se articula con un nuevo régimen de visibilidad de aquellas que antes se encontraban solapadas.

En el área metropolitana de Buenos A ires, esa situación se refleja en la coexistencia de medicinas tradicionales (que incluyen el corpus de saberes y prácticas del curanderismo y del chamanismo), medicinas religiosas (es decir, los distintos cultos y rituales terapéuticos inherentes a distintas cosmologías como las del catolicismo, las religiones afrobrasileñas, las diferentes denominaciones evangélicas, entre otras), medicinas 'alternativas' (las cuales remiten al conjunto de prácticas y saberes comúnmente asociados al movimiento new age y que incluyen disciplinas como el yoga, el reiki, el tai chi chuan, la aromaterapia, la cromoterapia, las terapias de vidas pasadas, la acupuntura, la digitopuntura, la reflexología, entre muchas otras), y el autotratamiento ${ }^{1}$.

A I mismo tiempo, como bien indica Singer (1990, p. 183), el estudio de ese fenómeno exige reconocer el hecho de que la naturaleza de los vínculos establecidos entre estos dispares enfoques médicos supone una serie de tensiones y pugnas (a nivel epistemológico y político) en el marco de las cuales no puede dejarse de lado el lugar que ocupan las formas científicamente legitimadas de cuidado, lideradas por la biomedicina. Es por ello que el estudio de estos abordajes terapéuticos no-biomédicos, más que reducirse a sí mismos, exige el desarrollo de un enfoque que contemple las características de su inserción en esta compleja red de relaciones, donde se definen las especificidades, las transacciones de sentido y las exclusiones que hacen a la construcción de toda identidad social.

A la luz de lo esbozado, el presente trabajo tiene como objetivo el análisis de la lógica de constitución de una identidad terapéutica en particular: aquella que remite a los diversos enfoques incluidos en el universo simbólico de las denominadas 'terapias alternativas'. Para ello, tomaremos como referente teórico el debate actual que plantean las teorías postestructuralistas que conjugan elementos de la lingüística (reconociendo, no obstante, que los conflictos desatados en el campo de la salud trascienden ampliamente la lógica del discurso), lo que nos permitirá destacar la dimensión relacional a partir de la cual esa identidad se constituye de modo negociado, es decir, aparece como el resultado de procesos que suponen el carácter flexible, nunca predeterminado y en constante diálogo de esa formación discursiva respecto a otros relatos que se orientan a la gestión de los procesos de salud/ enfermedad.

Para una mayor claridad expositiva, el presente trabajo se encuentra dividido en dos partes. La primera busca identificar los rasgos principales de la configuración del campo de atención de la salud, tomando como eje conceptual la distinción entre identidades hegemónicas/ identidades subalternas. En un segundo apartado, delinearemos algunos elementos que hacen a la lógica de construcción de la identidad terapéutica propuesta. Aquí se retomarán algunos postulados esbozados por Ernesto Laclau en diferentes trabajos académicos, especial mente los relativos a la construcción de las identidades sociales - equivalencia, antagonismo y hegemonía ${ }^{2}$ - y otros conceptos como el de plenitud, que permite pensar la operación simbólica a partir de la cual un particular es elevado a universal, haciendo posible la representación de un objeto "que, por definición, trasciende toda representación" (Laclau, 2002, p. 36). A través de esos conceptos y de la lógica de construcción de la identidad social que subyace a ellos, intentaremos conceptual izar la especificidad identitaria del universo terapéutico alternativo contemplando, al mismo tiempo, los elementos posibles que hacen a la heterogeneidad que presentan en los diversos ámbitos en los que se ejerce su práctica.

A pesar del carácter sustancialmente teórico de este trabajo, cabe destacar que las interpretaciones realizadas se fundamentan en materiales originales que construimos en el marco de un proyecto de investigación de tesis doctoral orientado al análisis de las representaciones y experiencias que los sujetos esbozan en torno a la enfermedad, la salud y la cura en el contexto de una terapia alternativa particular: la reflexología, proyecto que cuenta con financiamiento CON ICET desde 2007. De acuerdo con ese foco de interés, la estrategia metodológica instrumentada se ajusta a los criterios de abordaje de la investigación cualitativa, teniendo en cuenta la perspectiva de Fredrik Barth (2000), desarrollada para el abordaje etnográfico de

1 Recuperamos aquí la clasificación realizada por la antropóloga Idoyaga Molina (2002), de acuerdo a la cual funciona, en el Área Metropolitana de Buenos Aires, un sistema de atención de la salud caracterizado por el traslapo (overlap) de la biomedicina y los abordajes médicos señalados en el texto. Esa perspectiva retoma el concepto de sistema etnomédico propuesto por Good (1987) en el contexto de sus estudios en África. 2 En tanto núcleos conceptuales de su pensamiento, esas nociones atraviesan la totalidad de la obra de Ernesto Laclau. No obstante, es posible mencionar particularmente Laclau (1996) y Laclau y Mouffe (2004). 
mundos socioculturales que exceden el formato tradicional del estudio antropológico de la "comunidad". En efecto, de acuerdo a los itinerarios terapéuticosque hemos podido reconstruir (tanto en usuarios como en alumnos y especial istas) consideramos heurísticamente productivo realizar una lectura de esa terapia en términos de un lugar "de cruce", un eslabón en una cadena más amplia de vínculos con otras terapias, lo que metodológicamente permitió el establecimiento de relaciones significativas en pos de dar cuenta de las formas de circulación y de construcción de identidades al interior de ese universo. Es por ello que, más que circunscribir el análisis a los aspectos que hacen al universo de creencias y prácticas de la reflexología como si se tratara de un universo "cerrado", nos propusimos su abordaje en términos de un campo de diversidad (Barth, 2000, p. 192), lo cual supone su inscripción en una red de relaciones que involucra personas y formas institucionales diversas, capaces de vehiculizar distintos abordajes terapéuticos y distintas combinaciones entre los mismos, según las creencias a las que adhiera cada especialista en particular. C abe destacar por último que - en concordancia con el enfoque cualitativo adoptado - se utilizó la observación participante en diferentes cursos, workshops y clases de formación, así como entrevistas abiertas, extensas y reaurrentes a usuarios, alumnos y especialistas de reflexología, en tanto consideramos esas técnicas como los instrumentos de registro más acordes al método etnográfico (Griaule, 1969; H ammersley y Atkinson, 1994).

\section{Contexto general: la relación biomedicina - terapias periféricas en el marco de los sistemas oficiales de atención de la salud}

Diversos estudios contemporáneos se han focalizado en el problema de cómo los distintos saberes y prácticas médicas formulan la realidad en términos distintivos. Bajo ese horizonte de análisis, se destaca una profusa producción académica que se ha centrado en el estudio de la biomedicina, considerando el lugar clave que la misma ha ocupado en la configuración histórica de la modernidad y de sus instituciones, existiendo un consenso en lo que concierne a su base epistemológica y las consecuencias que de ella se derivan. Como ha señalado Luz (1997), la racionalidad biomédica aparece asociada a una cosmología analítica que se articula con el imaginario mecani- cista de la física de los siglos X VI y XVII. A partir de esta matriz de interpretación que ve a la dolencia como una entidad concreta, provocando lesiones que deben ser buscadas en el organismo físico, se consolida el dualismo que atravesará a la disciplina (formación, práctica profesional) y determinará una relación de subordinación/ exclusión respecto a otros paradigmas médicos: esta es la escisión entre el cuerpo - materia - y el hombre - espíritu. Si bien sería erróneo afirmar que la biomedicina constituye un corpus de nociones y prácticas influenciadas unívocamente por los modelos y/ o conceptos teóricos de la física o la biología (lo que terminaría delineando al campo biomédico como una totalidad homogénea sin fisuras) ${ }^{3}$, es necesario reconocer el papel que esta mirada dualista va a jugar en el rechazo de la medicina oficial hacia las terapias no- científicas, lo que determinará la atribución de la categoría de creencia a todo tipo de comprensión de la enfermedad cuyas referencias empíricas no sean claramente identificadas (Good, 1994).

Esta epistemología empirista se constituye en uno de los pilares que permitió legitimar las pretensiones de totalización de las formas de intervención sobre la salud de las poblaciones, dando como resultado lo que M enéndez (1990) definió en términos del carácter hegemónico del modelo de atención de la salud biomédico, devenido en oficial hacia fines del siglo XIX en la mayoría de los países occidentales. Esto debe interpretarse como parte de un proceso que - como indica la literatura académica sobre el tema - se da en virtud de la convergencia de un modo específico de concebir la realidad corporal (legitimada a través de los grandes relatos de la ciencia) y su implementación a nivel de las estructuras de intervención estatales, lo que redundó en un fuerte disciplinamiento de la población (Tesser y Luz, 2008). A la vez, ese proceso supuso la persecución sistemática de las terapéuticas de índole tradicional (en Argentina, el curanderismo y los distintos shamanismos indígenas), así como la incorporación subsumida de saberes y prácticas específicas (tomemos aquí como ejemplo la constitución de la especialidad kinesiológica en el marco biomédico, proceso histórico que - como bien indica M cGuire (1988) para el caso estadounidense - involucró una doble dinámica de persecución y apropiación de saberes y técnicas, como las utilizadas por los bonesetters - hueseros -, por ejemplo).

A hora bien, es interesante resaltar que, en las últimas décadas, se registra una serie de transformaciones que operan un giro en esa lógica, a partir de la cual se han delineado de modo dominante las políticas públicas y la organización institucional de la atención de la salud occidentales. En términos generales, esos cam- 
bios se traducen en una tendencia discursiva global que enfatiza la necesidad de combinación de la práctica biomédica con terapias y enfoques médicos excluidos del régimen oficial - habitualmente definidos bajo la denominación de 'medicinas alternativas y complementarias' (M AC) o 'no-convencionales'. Si bien este proceso de cambio se encuentra lejos de trastocar en términos reales las estructuras de poder establecidas en el actual campo de atención de la salud en la mayor parte de los países occidentales (Sacks, 2001) - en la medida que la medicina ortodoxa continúa monopolizando la gestión institucional, la distribución de recursos y la legitimidad simbólica - ese fenómeno se hace cada vez más presente en la producción de documentos de organismos internacionales que fijan estándares de normatividad para los gobiernos nacionales (O M S, O PS) ${ }^{4}$, en la emergencia paralela de asociaciones y ONGs con objetivos 'integrativos' y, de modo notable, en la proliferación de esas prácticas en los 'intersticios' de las instituciones hospitalarias oficiales (sobre todo en A rgentina, donde las medicinas otras no cuentan con ningún tipo de reconocimiento jurídico o estatal).

Siguiendo el vocabulario esgrimido por Foucault (2002), ese movimiento discursivo adquiere especial relevancia en la medida en que plantea la pregunta en torno a la posible emergencia de un nuevo umbral epistemológico en lo que hace a la relación de la biomedicina y las terapias no-científicas. D esde una perspectiva macrosocial, esa búsqueda por 'integración' puede ser tematizada, tal como precisa Easthope (1993), como una estrategia relativamente novedosa delineada desde las instituciones médicas oficiales para enfrentar la creciente diversificación del campo de la salud y la mayor aceptación por parte de los legos de terapias no-biomédicas, habilitando así a una incorporación selectiva de estas últimas bajo una lógica de subordinación (Easthope, 1993) o de domesticación (Fadlon, 2005). Esto último involucra un proceso de apropiación institucional de técnicas propias de las terapias holísticas en la práctica de fisioterapeutas biomédicos (C ariello M oraes, 2006; Goldstein et al., 1985). U n caso paradigmático en diferentes países es la reglamentación de la acupuntura como práctica clínica oficial que sólo puede ser realizada por biomédicos.

Por otra parte, el análisis relacional nos habilita, asimismo, al estudio de las dinámicas propias que se gestan desde la perspectiva de las terapias que ocupan un lugar periférico en el sistema oficial de atención de la salud. En ese sentido, es preciso tener en cuenta la propia capacidad de pugna, los recursos puestos en juego y las estrategias desarrolladas por cada agente social en particular. Es aquí donde podemos mencionar las acciones vehiculizadas por los agentes que representan a los distintos enfoques terapéuticos que entran en competencia entre sí, así como las acciones emprendidas por los legos, quienes devienen en usuarios de estas ofertas, formulando demandas de salud específicas sobre la base de acervos socioculturales definidos que las sustentan.

De ese modo, y lejos de una idea estática, ahistórica e inmutable respecto a lo que constituye una 'posición subalterna' en el campo de la salud, se impone el estudio de las modalidades en que ésta se actualiza de modo diferencial, a partir de la definición identitaria que delinean en su discurso los propios agentes implicados. C omo señala Spivak (2003), el concepto teórico de subalternidad adquiere relevancia en tanto hace blanco en la posición estructuralmente más débil en el marco de los discursos que en un momento histórico determinado configuran la textualidad social. Es por ello que, en la lucha por el reconocimiento, el subalterno virtualmente se encuentra 'silenciado' no por una ausencia literal de voz, sino más bien debido al lugar periférico de enunciación que ocupa, constituyéndose así en una figura de la diferencia radicalizada en términos de alteridad. A hora bien, aunque consideramos que las terapias 'alternativas' ocupan efectivamente una posición discursiva marginal en relación al carácter hegemónico del discurso biomédico, cabe analizar cómo las terapias alternativas ponen en cuestión 0 , por el contrario, contribuyen a reproducir esa hegemonía. Del mismo modo, es preciso observar de qué modo se relacionan con otros abordajes terapéuticos como, por ejemplo, las medicinas tradicionales de A rgentina, las cuales aparecen asociadas a sectores de la sociedad fuertemente marginados tanto desde un punto de vista de clase como étnico ${ }^{5}$, ocupando un lugar subordinado en términos diferentes que las 'alternativas'.

Es en ese marco que consideramos el potencial heurístico de la recuperación de los aportes real izados desde la teoría contemporánea de la hegemonía, centrándonos en el modelo desarrollado por Laclau para el estudio de las identidades. La elección de ese autor se funda en las posibilidades analíticas que ofrece para distinguir losalineamientos con otros discursos médi$\cos$ (lo que se verifica en las operaciones de apropiación de elementos compartidos con paradigmas disímiles), así como los juegos de exclusiones y reconversiones de sentido que este enfoque pone de manifiesto.

\footnotetext{
4 Respecto a la OMS, nos referimos a las recomendaciones que esboza en el documento "Estrategia sobre Medicina Tradicional" (2002- 2005) en el cual enfatiza el crecimiento de la demanda de medicinas no-alopáticas, tanto en países desarrollados como "en desarrollo". En cuanto a la OPS, se destacan las declaraciones que aparecen como resultado del / Taller Continental sobre los Pueblos Indigenas y la Salud, Winnipeg, Canadá. 5 Aunque diversos estudios empíricos han demostrado el uso de medicinas tradicionales en el seno de diferentes sectores socioeconómicos, incluyendo las clases altas. Al respecto, véase Idoyaga Molina (2002).
} 


\section{Equivalencia, antagonismo y hegemonía en la construcción de las identidades: una mirada desde la teoría de Ernesto Laclau}

Como ya hemos indicado, uno de los problemas que se presentan a la hora de abordar analíticamente la temática médica es el de la construcción de una jerarquización ontológica que se traduce en una escisión tajante entre la dimensión objetiva (que incluye el estudio del cuerpo, considerado como una serie de procesos físico-químicos) y la dimensión subjetiva del ser humano (a la cual le es asignado un estatus psicológico o bien se le relaciona con la idea de particularismo cultural) ${ }^{6}$. De ese modo, se actualiza en esta área específica del conocimiento el clásico dualismo entre naturaleza y cultura (par conceptual clave de la antropología y uno de los ejes centrales de la crítica feminista), el cual se caracteriza por proponer a la primera como el lugar que encierra la esencia y realidad última de toda identidad que tenga como soporte principal al cuerpo. Aquí, la propuesta teórica de Laclau constituye una contribución interesante en pos de resolver esa tensión, la cual pone en cuestión las interpretaciones esencialistas que conciben a cada paradigma terapéutico como una identidad autónoma y clausurada en sí misma. Como indica Good (1994), esto último aparece radicalizado en el caso de la biomedicina en virtud de su teoría nominalista del lenguaje, según la cual se considera que sus elaboraciones 'se desprenden' directamente de las observaciones del mundo natural.

U na de las claves que nos interesa resaltar a partir de esa problemática se sitúa en la lógica de constitución de las identidades sociales, las cuales aparecen en el marco del enfoque laclauniano como el resultado de un proceso de totalización hegemónica. En efecto, de acuerdo a la teoría de la representación de Laclau, en lo social no hay un plano extradiscursivo en virtud del cual resulte posible plantear una literalidad última de la realidad. Los objetos sociales (en nuestro caso particular los procesos de salud-enfermedad-atención) se encuentran investidos por una desbordante intertextualidad o polifonía de discursos que se disputan el derecho por la nominación legítima, en virtud de lo cual, más que de sentidos originarios, es menester hablar de una ausencia originaria de todo referente ( $L a-$ clau, 1996, p. 74). Es por ello que la pretensión de un 'acceso' a la realidad 'objetiva', negando la mediación de dispositivos discursivos para ello es, en sí misma, ideológica ${ }^{7}$. A hora bien, a pesar de que la complejidad de lo social lo torna por principio inexpresable, es evidente que la condición de posibilidad de toda fijación de sentido depende de la capacidad de constituir objetos plausibles de ser representados ya que, de lo contrario, no habría sentido en absoluto. Esto implica una operación de cierre del sistema de significación que, aunque ilusoria, funcione efectivamente como instancia de articulación unitaria de los elementos que conforman a esos objetos sociales.

Para pensar este proceso de totalización en el plano de la realidad social, el autor propone una reelaboración crítica del concepto gramsciano de hegemonía, focalizándose en la articulación de una serie de operaciones diferenciadas. ${ }^{8}$. La primera de ellas consiste en la construcción de una cadena de equivalencias que permita 'igualar' un conjunto de elementos particulares - y, por ende, diferentes entre sí - en torno a un significante vacío, es decir, un significante sin significado que represente la unidad de la cadena. Eso resulta posible en la medida en que ese significante dota de un horizonte de universalidad al conjunto, al articular cada uno de los particularismos en una red coherente de sentido. Sin embargo, no se trata de una mera agregación de elementos desarticulados: la función de universalidad siempre es encarnada por un objeto particular que, vaciándose parcialmente de su contenido, logra expresarse a sí mismo a partir del plus metafórico que resulta del establecimiento de la cadena equivalencial como totalidad (lo que implica una destrucción de rasgos diferenciales).

La segunda instancia tiene que ver con la formación de una frontera interna antagónica, lo que pone a la diferencia como punto de disrupción que bloquea la expansión de significantes y define los límites de la identidad. Ese punto reviste especial importancia ya que, en la medida en que el establecimiento del sistema de significación constituye el resultado de una pugna, los límites que termina definiendo no son neutrales, sino que tienen el carácter de una exdusión. En las palabras de Laclau (1996, p. 96), se trata de "un

6 A estas dos dimensiones se les asignan, además, estatutos de realidad diferenciales. En torno a los dualismos que atraviesan a los saberes y prácticas biomédicos (sobre todo en relación a la alopatía), ver Kirmayer (1988)

7 Aquí se vislumbra el modo en el que es recuperada la noción de sobredeterminación, introducida por Freud en sus escritos sobre los sueños y elaborada luego por Althusser. Según ese último autor, la sobredeterminación implica la ausencia de una ley social inmanente que fijaría momentos necesarios en la constitución de las relaciones sociales, frente a la cual lo simbólico se constituiría como plano de significación segunda y derivada. Sobre este tema, ver Laclau y Mouffe (2004, p. 142).

8 Respecto a esas tres operaciones, nos atenemos principalmente a lo esbozado en Laclau (2005), si bien en otros escritos (Laclau, 1996, 2002 y Laclau y Mouffe, 2004) se expresa como dialéctica de la encarnación/deformación, del universal/ particular o de la lógica de la equivalencia y la lógica de la diferencia. 
más allá que no es sólo una diferencia más sino algo que plantea una amenaza (es decir, que niega) a todas las diferencias interiores a ese contexto - 0 , más bien - que el contexto como tal se constituye a través del acto de exclusión de algo ajeno, de una exterioridad radical". N o obstante, el establecimiento de las fronteras identitarias no debe ser pensado en términos rígidos. Por el contrario, se trata de un concepto que habilita al análisis de las articulaciones hegemónicas en términos flexibles, debido a que los significantes flotantes se caracterizan por su capacidad de permitir la incorporación de elementos en principio externos a la cadena equivalencial ${ }^{9}$.

Por último, la tercera instancia consiste en la configuración de un sistema estable de significación, lo que remite a la necesaria reproducción de las operaciones simbólicas que brindan una cierta autonomía y permanencia a la estructura interna de la cadena articulada hegemónicamente. Esta instancia de reproducción no es nunca idéntica a sí misma, sino que introduce continuamente variaciones que ponen en juego la configuración de la identidad social. Eso implica que, si bien es posible incorporar elementos particulares que tensionan desde adentro la cadena equivalencial con nuevas diferencias, la unidad de la totalidad no se ve comprometida.

\section{La estrategia identitaria de las terapias alternativas: los momentos de la totalización hegemónica}

A pesar del carácter formalista e incluso logicista del planteo desarrollado, es posible retomar algunos de sus elementos a la manera de conceptos 'sensibilizadores', con el objeto de echar luz a los distintos momentos analíticos involucrados en la construcción de la identidad terapéutica alternativa. En este apartado nuestro propósito consistirá en desplegar una serie de mediaciones que habiliten a la aplicación de los conceptos mencionados a la interpretación de la construcción identitaria 'alternativa', con sus especificidades y matices.

En primera instancia, y siguiendo lo esbozado por Laclau, es de interés destacar el modo en el que el término 'alternativo' permite articular un espectro amplio y sumamente heterogéneo de prácticas, técnicas y saberes, que se unifican en una misma cadena de equivalencias. De ese modo, abordajes y técnicas que suponen no sólo diferentes contextos culturales de procedencia sino incluso de cosmologías - incluyendo terapias de origen occidental como la homeopatía, pero también otras de raigambre oriental que han sido profundamente refiguradas dando lugar a lo que algunos autores problematizan como un proceso de 'orientalización de occidente' (C ampbell, 1999; Silva da Silveira, 2005) - aparecen subsumidos bajo un nombre común, que es el que constituye su anclaje identitario. En ese marco, las nociones de 'energía' (que adopta distintos calificativos según la práctica que se tome como referencia: prana, chi o ki, rei, etc.), 'flujos energéticos', 'equilibrio/ desequilibrio', 'unidad cuerpo-mente', 'holístico', 'persona como totalidad', aparecen como significantes vaćos que irrumpen como el punto de máxima saturación del sentido, articulándose a una mixtura de símbolos (mantras, figura del yin/ yang, estatuillas de buda), objetos (cristales, velas, inciensos) e imágenes (de personas asumiendo distintas posturas - del yoga, del tai chi chuan o de meditación, lo que recurrentemente se retoma en la publicidad -, imágenes de campos energéticos, ideogramas orientales, entre muchas otras). Eso reviste especial relevancia en la medida en que permite a la cadena presentarse como algo más que sí misma, más allá del sentido literal que cada uno de los términos implicados efectivamente revisten, generando una suerte de plus metafórico que condensa la imposible plenitud de la idea de 'salud' en términos de 'armonía'10.

Lo antedicho nos permite vislumbrar que, por un lado, no es que las referencias constantes al logro del 'equilibrio' sean la expresión literal de un aspecto exclusivo (y excluyente) de la identidad alternativa, ya que claramente esas ideas son compartidas por otros abordajes médicos e incluso desde algunas ramas de la medicina científicall. Por el contrario, la identidad que unifica al abigarrado universo alternativo se configura retroactivamente, al reconocerse cada parte integrante del mismo en esos símbolos y prácticas, al verse representado por ellos. Por otro lado, el lugar central que asume la simbología no-verbal en la constitución de la identidad terapéutica alternativa nos permite comprender de modo más acabado la 'ausencia de palabras'

\footnotetext{
9 Eso implica que el significante vacío ('alternativo', 'salud', etc.) puede expresarse a sí mismo a través del plus metafórico que se genera como resultado de su deformación (equivalenciación con otros significantes) y el establecimiento de un exterior constitutivo.

10 Esos símbolos, objetos e imágenes suelen representar a las 'terapias alternativas' como un todo, particularmente en el marco de la publicidad (sea de los institutos que ofertan esas prácticas, sea en los medios masivos de comunicación -donde se busca mostrar un producto pasible de contribuir a ese logro de 'armonía').

11 Como indica Laplantine (1999, p. 64-76), diferentes corrientes médicas occidentales se inscriben en formas de interpretación del padecimiento de índole relacional, eso es, que consideran a la enfermedad no tanto como una entidad enemiga y extraña, sino como un desarreglo o desequilibrio. El autor menciona la medicina hipocrática, la patofisiología, el vitalismo médico del siglo XIX, la homeopatía y el psicoanálisis. Así, la idea de equilibrio no es privativa de las "alternativas", encontrándose también en el centro de diferentes corrientes de la biomedicina.
} 
que registramos en reiteradas oportunidades a la hora de interrogar a nuestros informantes acerca de los factores que los condujeron a abrazar ese tipo de terapias. Eso nos remite al concepto de plenitud mística (Laclau, 2002 , p. 36) que permite hacer referencia al modo en el que la representación del 'todo' se realiza a través de un objeto, situación o símbolo en particular.

Las características específicas de esa producción discursiva de la unidad suponen un giro relativamente novedoso en lo que hace a la nominación de las medicinas no- científicas, lo que es a nuestro entender de especial interés a la hora de evaluar los efectos de legitimación que esas terapias son capaces de generar frente a la sociedad civil y a la medicina oficial (al menos si tomamos como referencia al caso argentino). En ese sentido, si retomamos el estudio de Di Liscia (2002) sobre la consolidación institucional de la biomedicina en Argentina, la posición que se asume frente a los enfoques médicos periféricos aparece como compleja, registrándose diferencias importantes en las posturas asumidas por parte de los múltiples actores sociales involucrados en diversos momentos históricos (funcionarios estatales, eclesiásticos, viajeros, médicos y periodistas, entre otros). No obstante, la autora muestra cómo, en última instancia, el discurso oficial termina por articular una división dicotómica entre los significantes 'medicina' y 'curan derismo', siendo esa última noción una etiqueta generalizante que se utiliza de modo sistemático cuando se trata de denominar a toda práctica no-científica. Bajo el término curanderismo, entonces, se engloba toda una serie de saberes susceptibles de ser descalificados, al establecerse una asociación directa con ideas como 'antihigiene', 'charlatanería' e 'ignorancia', lo que incluye las distintas medicinas indígenas primero $\mathrm{y}$, a partir del proyecto modernizador de 1880 , se centra en las prácticas empleadas por los multiformes 'sectores populares' que tienen como figura clave al inmigrante (D i Liscia, 2002 citado en Faberman y Tula M olina, 2005)12.

Por el contrario, la irrupción de las medicinas alternativas en ese espacio de relaciones desencadena otro tipo de lógica de representación de las terapias no-biomédicas, en el que puede registrarse una activa producción discursiva de la diferencia respecto a lo anteriormente señalado como 'curanderismo'. En efecto, por parte de los especialistas alternativos, ese distanciamiento oscila entre una exclusión directa del vocabulario y de las técnicas específicas de raigambre tra- dicional, o bien implica una inclusión solapada en sus discursos, que supone una reelaboración de las mismas ideas en relación al carácter holístico de la terapia, la enfermedad y la salud en términos diferenciales. Esa reelaboración comprende un amplio espectro de posibilidades: por un lado, es común constatar que la interpretación de taxones tradicionales (como lasfiguras del daño, el mal de ojo y la brujería) se lleve a cabo en términos alternativos - 'mala onda' 0 energías 'negativas', pasibles de ser transmitidas por contacto 0 a distancia (Saizar, 2007). Por otro lado, pudimos registrar la apropiación de algunas de sus técnicas terapéuticas, lo que implica en muchos casos la resemantización de las mismas en términos de prácticas chamánicas ${ }^{13}$, denotando así una estrategia retórica exotizante. De esa manera, podemos arriesgar a modo de sugerencia que se opera un desplazamiento discursivo que implica un alejamiento respecto de un corpus de nociones históricamente asociadas a 'lo popular' (aunque no necesariamente el fenómeno del curanderismo se circunscriba a esos sectores ${ }^{14}$ ), lo que puede ser comprendido si tomamos en cuenta que las 'alternativas' constituyen una oferta de costo considerable en la mayoría de los casos, siendo utilizadas principalmente por individuos pertenecientes a sectores de nivel socioeconómico medio/alto y de cultura específicamente urbana residentes en Buenos Aires.

Pues bien, como podemos observar, es clave tener en cuenta que la estructuración de la identidad terapéutica alternativa no sólo se lleva a cabo sobre la base de la exclusión de otros abordajes médicos al ser tematizados como incompatibles, sino que se fundamenta sobre to do en la tarea constante de apropiación y reelaboración de nociones diversas.

Esto remite al carácter siempre abierto e indeterminado de los significantes los cuales - lejos de suturarse de un significado fijo - mantienen un punto de apertura y conexión con los elementos discursivos que se encuentran del otro lado de la frontera identitaria. Lo antedicho es particularmente evidente en el universo simbólico de las terapias alternativas, el cual se caracteriza por su capacidad de ajuste 0 adecuación a los esquemas interpretativos propios de distintos estilos culturales o 'de pensar' (D ouglas, 1998). Eso se expresa en el hecho de que los significantes que forman parte del discurso alternativo son fácilmente 'exportables' a otros universos de sentido, al tiempo que aquel absorbe y retraduce elementos externos en sus propios términos

12 La denominación 'curanderismo' en los términos que acabamos de esbozar se distancian, claro está, de su definición en clave académica, teniendo que ver más con el modo en el que las prácticas tradicionales de Argentina son representadas en el imaginario biomédico y en el seno de algunos sectores de la sociedad.

13 Como ejemplo, podemos referir el caso de un curso dictado en diciembre de 2007 en uno de los institutos donde realizamos el trabajo de campo. El curso estaba intitulado "Sanaciones chamánicas" y en los panfletos que circulaban sobre el mismo se señalaba: "Siguiendo las antiguas tradiciones conocerán la forma de tratar: culebrilla, verrugas, pata de cabra, parásitos, empachos, mal de ojos, etc."

14 Distintos estudios documentan el uso de prácticas tradicionales por parte de sectores medios y altos, que pueden llegar a pagar sumas importantes de dinero a especialistas curanderos. Véase Idoyaga Molina (2002) 
- los cuales pasan a formar parte, entonces, del conjunto de significantes flotantes cuyo significado es fijado retroactivamente al pasar a formar parte del sistema.

$C$ omo ya hemos indicado en un trabajo de enfoque cualitativo (A Igranti y Bordes, 2007), eso se verifica en nuestro contex to de estudio particularmente en relación a la noción de 'energía', la cual es sumamente porosa a la hora de asignar significado a un amplio espectro de experiencias de enfermedad, sufrimiento, pero también de sanación y bienestar. Eso se registra en relación a usuarios pertenecientes a distintos estilos de pensar: se trate de una persona con un perfil claramente alternativo (contexto en el cual la energía es definida a partir de una cosmología orientalista), de una persona que detenta una adhesión más fuerte a las creencias de raigambre católica (en el marco de las cuales la energía 'alternativa' es homologada a la idea de 'fuerza espiritual'). 0 bien se trate de un perfil que opta por las explicaciones científicas, quienes pueden interpretar esa noción bajo los términos propuestos por la fisiología o la anatomía, retomando representaciones vinculadas al sistema nervioso, lo que involucra un amplio espectro de nociones que son equiparadas al flujo energético vislumbrado desde el ideario orientalista (en ese sentido, los 'estímulos' realizados en el marco de disciplinas como la acupuntura, la digitopuntura y la reflexología, por ejemplo, se 'desplazarían' por el cuerpo a través de músculos y nervios) ${ }^{15}$. A simismo, podemos consignar el caso de individuos que explican la dimensión energética de la corporalidad humana en términos de la física, principalmente retomando los postulados popularizados de la física cuántica ${ }^{16}$. Por último, es menester destacar la fuerte asimilación que las terapias alternativas han hecho del discurso psicoanalítico, refigurando profundamente nociones como las de inconciente, represión y negación, por ejemplo, aplicándolas a la interpretación de los procesos cuerpo-mente en términos holísticos, lo que ya ha sido señalado por M aluf (2005).

En relación a esta segunda operación de la totalización hegemónica de la identidad 'alternativa', sólo agregaremos que la mencionada capacidad de asimilar elementos que en principio tienen raigambre en otros paradigmas médicos habilita al desarrollo, tanto por parte de institutos (de formación y oferta de servicios) como de especial istas, de estrategias de legitimación disímiles. Estas se corresponden, según lo que hemos podido recabar en nuestro trabajo de campo en el universo terapéutico de la reflexología, con los objetivos que el ejercicio de la disciplina (y/o disciplinas) suponen para cada actor social en particular, lo que termina delineando perfiles identitarios específicos. Sin suponer una relación de mutua exclusión, podemos señalar distintos perfiles, entre los cuales encontramos una oferta más volcada a enfatizar los aspectos propiamente espirituales del discurso alternativo, lo que es asociado al movimiento de la N ueva Era y aparece bajo la forma de una forma de religiosidad difusa y descentralizada (A maral, 1999; C arozzi, 2001; Soneira, 2001, entre otros). Esa orientación impulsa, en muchos casos, a la utilización de la parafernalia alternativa para la oferta como un bien de consumo, lo que tiene como uno de sus elementos centrales el discurso centrado en el 'conócete a ti mismo'.

Por otra parte, en los casos en que los terapeutas buscan fortalecer el aspecto propiamente terapéuticomédico de la oferta, se observan dos estrategias posibles: 1) una que se caracteriza por un alineamiento discursivo con la biomedicina, llegando incluso a promover acciones concretas en la búsqueda de reglamentación y, así, el reconocimiento por parte del Estado de la práctica en cuestión; y 2) otra que se funda en criterios de legitimidad de corte tradicional en los términos de M ax Weber (1998), perfil que se cristaliza sobre todo en institutos de raigambre orientalista que predican la práctica de diversas disciplinas en el marco de la Medicina Tradicional China, o que se inscriben en un abordaje que tiene arraigo en la cosmología terapéutica de la cultura japonesa. Esos casos resultan interesantes, ante todo, debido a la relevancia que adquiere "el retorno a las fuentes" y el carácter de autenticidad que éste le imprime al saber adquirido constituyéndose, así, en el fundamento que garantiza la legitimidad de la práctica.

Como podemos observar de modo muy breve, el sistema de significación del universo alternativo habilita, en el marco de su heterogeneidad constitutiva, a la configuración de múltiples perfiles terapéuticos. En ese sentido, en contraposición a las lecturas simplistas que tienden a ver en las terapias alternativas una formación discursiva que homogéneamente se identifica con el ideario new age, es preciso enfatizar que hay una serie de elementos significantes más sutiles que se agregan a la cadena equivalencial y que se encuentran vinculados a contextos cosmovisionales diferentes. Es así que los límites móviles y constantemente redefinidos de esta identidad terapéutica - considerada en términos amplios - tienden a generar tensiones entre distintas escuelas, institutos e incluso entre distintas disciplinas respecto a la definición del auténtico 'ser alternativo' y sus implicancias en el ámbito de la práctica. Eso puede ejemplificarse con el caso de terapeutas que buscan identificarse con el costado más técnico y duro de la biomedicina, lo que incluso los impulsa a trabajar en

15 En el contexto de este trabajo de enfoque cualitativo, sólo pudimos registrar un discurso de incompatibilidad y exclusión: aquel relativo al ideario religioso del evangelismo.

16 Por supuesto, no queremos decir que los individuos que incursionan en el universo alternativo manejen el conocimiento experto de la física cuántica académica, sino que tienden a recuperar los saberes 'masificados' a través de la literatura de divulgación sobre el tema. 
ámbitos clínicos biomédicos (como, por ejemplo, en sectores de C uidados Paliativos de hospitales públicos). En nuestro trabajo de campo hemos podido constatar que los terapeutas pertenecientes a ese perfil pueden incluso llegar a manifestar su rechazo respecto a los saberes considerados 'esotéricos' como el reiki, en virtud del fuerte componente ritual y el uso de una simbología de corte religioso que reviste su terapéuti$\mathrm{ca}^{17}$. A simismo, en el vocabulario que vehiculizan esos agentes sociales, se constata una fuerte apropiación de los discursos institucionales de la medicina oficial, lo que se traduce en una constante referencia a su tarea en términos de práctica 'complementaria' - y no 'alternativa' - en relación a la biomedicina. Esa matriz interpretativa respecto a su práctica terapéutica se sustenta en la percepción negativa que ese término genera en los biomédicos, en tanto es interpretado como una propuesta que postula el carácter paralelo o excluyente de la medicina científica ${ }^{18}$. A partir de esa elaboración alternativa respecto a la actitud biomédica, uno de los efectos de sujeto que podemos hipotetizar es el de una construcción identitaria en dependenda, donde la complementariedad respecto a la biomedicina aparece bajo la forma de una subsunción.

Como dejamos entrever en el transcurso del trabajo, no obstante, esta virtual 'permeabilidad' de los discursos alternativos que les permiten ajustarse a distintas búsquedas y necesidades tanto por parte de terapeutas como de usuarios (así como también ajustarse a diferentes ámbitos institucionales) no redunda en un debilitamiento de la pertenencia al anclaje identitario 'alternativo' en lo que hace a sus postulados más estructurantes. Eso resulta posible en tanto los significantes vaćos inherentes a ese discurso operan como puntos nodales, esto es, como núcleos estables de significación que no sólo atraviesan sino que presiden toda producción de sentido (recordemos que en virtud de su función de nombrar una realidad imposible de representar, los significantes vacíos rompen su contigüidad con los otros significantes e irrumpen como el punto de máxima saturación del sentido, constituyéndose en la instancia que 'fija' el significado de los elementos flotantes). Se genera así un proceso retroactivo de producción de significado que se actualiza constantemente, en el que la formación discursiva mantiene la unidad más allá de que cada nuevo eslabón externo produzca un deslizamiento de sus fronteras ${ }^{19}$.
Siguiendo con este razonamiento, la preeminencia que tienen los significantes 'energía', 'armonía', 'unidad cuerpo-mente-espíritu', o la idea de tomar al paciente como una persona 'total', tiene fuerte implicancias en el discurso y en la práctica alternativa, marcando diferencias a veces irreconciliables con el discurso biomédico en relación a tópicos específicos como, por ejemplo, en lo que hace a la comprensión del paciente $(y$, por ende, en la definición de la relación médico-paciente). Al mismo tiempo, se verifican líneas de continuidad con otros abordajes terapéuticos que también tienen como objetivo del tratamiento el logro de la sanación desde un punto de vista integral u holístico, lo que desde la antropología puede ser leído como una búsqueda de conciliación entre las entidades que conforman a la persona en tanto tal ${ }^{20}$.

Es en la dimensión donde el discurso alternativo se reconoce como una unidad al interior de sus fronteras identitarias (gracias al carácter estable e irrevocable de ciertos tópicos clave) que ubicamos las tensiones que se dan entre, por ejemplo, biomédicos y terapeutas que consienten su permanencia en un lugar subordinado respecto a la biomedicina. Esa aparente ambivalencia (que se expresa en la búsqueda de legitimación frente a la medicina oficial, pero que a la vez sitúa un antagonismo constitutivo respecto a la forma de accionar cientificista) adquiere sentido si comprendemos que uno de los más interesantes aportes de la propuesta de Laclau es el carácter constantemente negociado de las identidades sociales, cuyas posiciones se redefinen y son actualizadas en contextos históricos, institucionales y culturales determinados. A pesar de que para el autor la instancia del antagonismo (que remite a las identidades no-equivalenciales que quedan por fuera de la frontera identitaria y aparece como el terreno del $\mathrm{O}$ tro) constituye la condición de posibilidad de la constitución de una identidad, recordemos que nunca se trata de una relación 'objetiva', sino la expresión de la imposibilidad de sutura última, de constitución plena de las identidades como totalidades empíricas inmutables (Laclau y M ouffe, 2004, p. 169). De ahí que - si bien a primera vista las terapias alternativas parecen instituirse en tanto una propuesta antagónica respecto a los supuestos teóricos y prácticos de la biomedicina, desde sus propios fundamentos identitarios (definidos en virtud de intereses profesionales -, de aspiraciones políticas y estrategias de legitimación) pueden buscar

17 Acerca de las instancias rituales de esa disciplina, véase por ejemplo Saizar (2005) y Babenko (2003).

18 Las referencias al 'horror' de los biomédicos hacia el término 'alternativo' (como nos indicó un especialista) y la consecuente preferencia del término 'complementario' por parte de los terapeutas, han sido recabadas a través de charlas informales, debido a la negativa de los informantes de ser registrados al hacer esos comentarios.

19 En ese sentido, se pone de manifiesto el modo en el que la nominación constituye retroactivamente su referencia, es decir, es el significante vacío el que, en el acto de nominar, produce un efecto perfomativo de coherencia de conjunto, 'haciendo' existir aquello que nombra.

20 La noción de persona constituye una categoría clásica de la antropología, y remite al conjunto de entidades -mente, alma, espíritu, entre muchas otras- que en un marco sociocultural determinado permite definir al ser humano. Al respecto, véase Marcel Mauss (1979). Por otra parte, la concepción de enfermedad definido como el desequilibrio de las entidades que conforman a la persona es señalado por Idoyaga Molina (2002). 
capitalizar el carácter 'flotante' de al gunos de sus significantes para identificarse con este enfoque hegemónico, a la vez de distanciarse del mismo, de acuerdo a la temática que se trate cada vez. Podemos señalar aquí la adhesión al discurso científico cuando se trata de enfatizar el carácter empírico de su eficacia terapéutica. Sin embargo, es innegable su rechazo cuando se trata, por ejemplo, de definir la práctica alternativa en términos de 'arte de curar', en contraposición al aspecto técnico $y$, por ende, cosificante del paciente en la biomedicina. Eso denota una mirada holista de la persona que es constitutiva de la relación terapeuta/ sujeto de cura y es central en la definición de la identidad alternativa.

\section{Palabras finales}

En el presente trabajo, hemos intentado realizar una contribución de corte conceptual al análisis del fenómeno emergente de las terapias alternativas, el cual constituye un verdadero desafío para la sociología en virtud del carácter peculiar de su conformación institucional (marcada por la heterogeneidad de sus orientaciones teórico-prácticas y su carácter descentralizado), el hibridismo cultural que revisten, así como la multiplicidad de modalidades de adhesión que asumen sus especial istas y usuarios. En ese contexto, buscamos analizar este objeto de estudio desde una perspectiva que privilegiara su dimensión relacional, esto es, en el marco de la red de relaciones que se configura en el espacio social de la salud. Aquí, a través de un marco conceptual de corte postestructuralista, intentamos observar las implicancias de la irrupción de esas nuevas ofertas terapéuticas en un contexto marcado por la posición hegemónica de la biomedicina. En ese sentido, nos interesó destacar que, si bien resulta incontestable el carácter subalterno o periférico que esas terapias revisten en el sistema oficial de atención, éstas cristalizan una serie de desplazamientos discursivos a nivel global (cuyo caso paradigmático es el de la O M S y la O PS), a la vez que condensan una serie de reclamos por parte de losusuarios, poniendo de relieve la fuerte injerencia del plano cultural en cuestiones vinculadas a la salud, a través de las críticas que desde diversos sectores se alzan hacia el modelo único de atención.

Debido a este lugar - al menos en parte - 'contestatario' que las terapias alternativas parecen ocupar en la actualidad, decidimos situarnos en el segundo apartado, desde un punto de vista que nos permitiera desplazar analíticamente al campo de la salud como espacio de estructuración de posiciones hegemónicas/ subalternas, a la propia perspectiva sustentada por los agentes que se inscriben en ese espacio terapéutico. De esa manera, se buscó reconstruir el modo en el que la posición periférica que ocupan es reactualizada y, en cierto sentido, reproducida en sus propios términos. Es por ello que tratamos de poner en evidencia la existencia de distintas estrategias de identificación y legitimación, definidas a partir de la elaboración diferencial de tres operaciones de constitución identitaria. Así, nos esforzamos en distinguir algunos criterios estructurantes del universo simbólico alternativo, en virtud de los cuales se llega a una articulación hegemónica que tiene como principio de inteligibilidad una serie de significantes específicos, como los de 'armonía', 'equilibrio', 'unidad' y 'holismo'. A la vez, el marco conceptual empleado nos permitió reconocer las operaciones en las que la dimensión de 'negociación' con la biomedicina y/ o con otros abordajes terapéuticos son centrales, poniendo en primer plano la importancia de la función que cumplen los significantes flotantes en la lógica de construcción de las identidades. De ese modo, resaltamos cómo el carácter indefinido de esos significantes es pasible de habilitar a que el discurso 'alternativo' se adapte al lenguaje y a la estructura relativos a diversos sectores sociales, que adhieren a distintos abordajes terapéuticos y que vehiculizan vocabularios acordes respecto de lo que es la salud, la enfermedad, y cómo debe efectuarse la curación.

En virtud de esta naturaleza 'doble' (esto es, a la vez periférica y desafiante) que esas terapias ocupan de cara a las relaciones de dominación estatuidas en el campo institucional de la salud, se plantea la pregunta acerca de si cuentan efectivamente con las características necesarias para constituirse en un sujeto político, con posibilidades de proponer un proyecto de atención y cuidado diferente al que es sustentado por la biomedicina en la actualidad. C omo indica Laclau (2008, p. 24-29), el proceso de construcción de un sujeto político es definido como parte de una transformación de los 'pedidos' en 'exigencias', en demandas movilizantes que contribuyan a crear la identidad del grupo a partir del proceso de definición de un enemigo común estableciendo, de ese modo, una frontera antagónica estable. Si bien a nuestro entender esa categoría no se aplica en esa acepción a nuestro objeto de estudio - ya que la constitución de un enemigo no llega a ser tan fuerte como para definir un grado de cohesión a un nivel de movimiento - es posible identificar, no obstante, la presencia de una dimensión política de ese objeto. Esa dimensión, vinculada con la puesta en evidencia de ciertas problemáticas, que en muchos casos son originadas a partir de la propia racionalidad biomédica ${ }^{21}$, constituye uno de los aspectos a ahondar en investigaciones empíricas futuras, ya que ha sido delineado aquí desde una perspectiva prominentemente conceptual. 


\section{Referencias}

ALGR AN TI, Joaquín; BOR DES, M ariana. Búsquedas de tratamiento de la enfermedad y la aflicción. A proximaciones al estudio de las estrategias de salud en usuarios de terapias alternativas y creyentes pentecostales. In: ID OYAGA M O LIN A, A. (O rg.). L os caminos terapéuticos y los rostros de la diversidad. Buenos A ires: Ediciones IU N AÁ rea T ransdepartamental de Folklore/ CAEA-CON ICET, 2007. [Tomo I].

AM AR AL, Leila. Sincretismo em movimento: 0 estilo nova era de lidar com o sagrado. In: CAR OZZI, M. J. (O rg.). A nova era no M ercosul. Petrópolis: Vozes, 1999.

BA BEN KO, Paula de Campos. 0 nde o paciente torna-se o curador: uma etnografia dos espaços terapêuticos rei kianos. V R EUNION DE ANTROPOLOGIA DEL MERCOSU R (R A M 2004). Florianópolis: U niversidade Federal de Santa C atarina, 2003.

BARTH , Fredrik. M etodologias comparativas na análise dos dados antropológicos. In: LASK, T. (O rg.) 0 guru, 0 iniciador e outras variaçóes antropológicas. $R$ io de Janeiro: C ontra C apa, 2000.

BO U R DIEU, Pierre. C osas dichas. M éxico: Gedisa, 2004. CAM PBELL, Colin. The Easternisation of the West. In: $\mathrm{N}$ ew religious movements: challenge and response. Londres: R outledge, 1999.

CAR IELLO MOR AES, M aria R egina. Práticas de acupuntura no contexto brasileiro. In: XI CONGRESSO LATIN O -AM ER ICANO SOBR E R ELIGIÃO E ETN I$C I D A D E$. A nais do $X I C$ ongresso $L$ atino-A mericano sobre $R E$ ligião e E tnicidade. São Bernardo do C ampo: U niversidade M etodista de São Paulo, 2006.

CAROZZI, M aría Julia. N ueva era y terapias alternativas: construyendo significados en el discurso y la interacción. Buenos A ires: Ediciones de la U niversidad C atólica, 2001. DI LISCIA, M aría Silvia. Saberes, terapias y prácticas médicas en A rgentina (1750-1910). M adrid: C SIC , 2002.

DOU GLAS, M ary. Estilos de pensar. Barcelona: Gedisa, 1998.

EAST HO PE, G ary. The response of orthodox medicine to the challenge of alternative medicine in Australia. A ustralia and $N$ ew $Z$ ealand Journal of Sociology, v. 39, n. 3, p.289-301, 1993.

FABER M AN, Judith; TU LA M O LIN A, Fernando. Reseña: M aría Silvia di Liscia, Saberes, terapias y prácticas médicas en A rgentina (1750-1910). R evista del Instituto de $\mathrm{H}$ istoria A rgentina y A mericana 'D r. E milio R avignani', n. 27, p.149-153, 2005.

FA DLO N, Judith. N egotiating the holistic turn: the domestication of alternative medicine. A lbany, N Y : State U niversity of $\mathrm{N}$ ew York, 2005.

FO U CAU LT, M ichel. L as palabras y las cosas. Buenos A ires: Siglo X X I Editores, 2002.

GOLDSTEIN , M ichael S.; JAFFE, D ennisT.; GAR ELL, Dale; BER KE, R uth Ellen. Holistic D octors: becoming a non-traditional medical practitioner. Journal of $C$ ontempora- ry E thnography, n. 14, p. 317-344, 1985.

GOOD, Byron. M ediane, rationality and experience. Cambridge: C ambridge U niversity Press, 1994.

GO O D, C harles. E thnomedical systems in A frica. N ew York: The Guilford Press, 1987.

GR IAU LE, M arcel. EI método de la etnografía. Buenos A ires: N ova, 1969.

H A M M ER SLEY, M artín; AT K IN SO N, Paul. E tnografía. Barcelona: Paidós, 1994.

IDOYAGA MOLINA, A natilde. C ulturas, enfermedades y medianas: reflexiones so bre la atención de la salud en contextos interculturales de Argentina. Buenos Aires: Prensa del IU N A, 2002.

KIR M AY ER, Laurence. M ind and body as metaphors: hidden values in biomedicine. In: LOCK, M.; GORD O N , D. (Eds.). B iomediane examined. D ordrecht: K luwer A cademic, 1988.

LAC LAU, Ernesto. E mancipación y diferencia. Buenos A ires: A riel, 1996.

M isticismo, retórica y política. Buenos A ires: Fondo de Cultura Económica, 2002.

- La razón populista. Buenos A ires: Fondo de Cultura Económica, 2005.

D ebates y combates. Buenos A ires: Fondo de Cultura Económica, 2008.

LACLAU, Ernesto; M O U FFE, Chantal. H egemonía y estrategia socialista: hacia una radicalización de la democracia. Buenos A ires: Fondo de Cultura Económica, 2004.

LAPLANTINE, François. A ntropología de la enfermedad. Buenos A ires: Ediciones del Sol, 1999.

LU Z, M adel. N atural, racional, social. Buenos A ires: Editorial Lugar, 1997.

. Cultura contemporânea e medicinas alternativas: novos paradigmas en saúde no fim do século $X X$. Physis: R evista de Saúde C oletiva, n. 15 (suplemento), p. 145-176, 2005.

M A LU F, Sônia. M itos coletivos, narrativas pessoais: cura ritual, trabal ho terapêutico e emergência do sujeito nas culturas da 'N ova Era'. M ana, v. 11, n. 2, p. 499-528, 2005.

M AU SS, M arcel. Sociología y antropología. M adrid: Editorial Tecnos, 1979.

M CGUIRE, M eredith. $R$ itual healing in suburban A merica. N ew Brunswick: R utgers U niversity Press, 1988.

M EN EN DEZ, Eduardo. M orir de alcohol. M éxico: Ediciones de la C asa C hata, 1990.

O MS (O rganización M undial de la Salud) Estrategia de la OMS sobre medicina tradicional (2002-2005). Disponible en: <http:// apps.who.int/medicinedocs/ es/d/Js2299s/ >, 2002. Acceso en: 15 jun. 2008.

O PS (O rganización Panamericana de la Salud) Informe del taller de medicinas y terapias tradicionales, complementariasy alternativas en las A méricas: políticas, planes y programas. D isponible en: <http:// w w w. paho.org/Spanish/AD / TH S/ O S/ 42-IN DI-span.doc>. Acceso en: 10 abril 2009. 
SAIZAR, M ercedes. Cuando el Dr. U sui meditó en el M onte Kurama. U na aproximación al análisis del mito de origen del reiki usui en Buenos Aires (A rgentina). M itológicas, n. 20, p. 41-58, 2005.

Elecciones diferentes y una búsqueda común. La selección de terapias en sectores medios y urbanos de Buenos Aires. In: L os caminos terapéuticos y los rostros de la diversidad. Ediciones IU N A-Á rea Transdepartamental de Folklore/ CAEA-CONICET, 2007. [Tomo I].

SAKS, M ike. A Iternative medicine and the health care division of labour. Present trends and future prospects. $C$ urrent Sociology, n. 49, p. 119-134, 2001.

SILVA DA SILVEIR A, M arcos. N ew Age \& neo-hinduísmo: uma via de mão dupla nas relações culturais entre 0 cidente e
O riente. C iendas Sodiales y R eligión, n. 7, p.73-101, 2005.

SINGER, M errill. Reinventing medical anthropology: toward a critical realignement. Social Sciences and M edicine, vol. 30, n. 2, p. 179-187, 1990.

SO N EIR A, Jorge. Ciencias Sociales y religión en el C ono Sur de A mérica Latina: diez años después (1991-2000). C iencias Sociales y R eligión, año 3, n.3, p. 143-149, 2001.

SPIVAK, G ayatri. ¿Puede hablar el subalterno? R evista C 0lombiana de A ntropología, n. 39, p. 297-364, 2003.

TESSER, Charles Dalcanale; LU Z, M adel. R acionalidades médicas e integralidade. C iência e Saúde C oletiva, v. 13, n. 1, p. 195- 206, 2008.

WEBER, M ax. E conomía y sociedad. M éxico: Fondo de Cultura Económica, 1998.

\title{
Analysis of the construction of "alternative" therapeutical identity in the context of the field of health in Buenos Aires
}

\begin{abstract}
In this article the author presents some conceptual elements for the analysis of alternatives therapies, usually related to the $\mathrm{N}$ ew A ge movement, focusing on the idea of the health field and the construction of collective identities. As regards this latter objective, the text introduces the theory of hegemony developed by Ernesto Laclau, in order to explore how alternative identities are built through operations of equivalence, reconversion and exclusion of some 'signifiers' that belong to the discursive formations of different medical approaches. The author reaches the conclusion that the flexible characteristics of this identity option allow the development of distinctive legitimating strategies for the specialists. This is a key to tackle the identity heterogeneity of this symbolic universe. This "flexibility" presents, at the same time, the analytical field to discuss the potential that alternative identities have to subvert or reproduce the dominant logic impose, in the field of health, by biomedicine or scientific medicine.
\end{abstract}

Keywords: alternative therapies; collective identities; Laclau; Buenos A ires.

\section{Análise da construção da identidade terapêutica "alternativa" no contexto do campo da saúde em Buenos Aires}

\begin{abstract}
Resumo
Este artigo apresenta alguns elementos conceituais para a anál ise das terapias alternativas, habitual mente associadas ao movimento $\mathrm{N}$ ew A ge, tomando como conceitos centrais a ideia de 'campo da saúde' e a construção de 'identidades coletivas'. A respeito desse último ponto, o texto se centra na teoria da hegemonia elaborada por Ernesto Laclau, o que permite indagar como a identidade alternativa se constrói em termos distintivos, a partir de operações de equivalenciação, reconversão ou exclusão de determinados significantes (que pertencem às formações discursivas de outros enfoques médicos). C hega- se à conclu são de que as características próprias dessa proposta identitária habilitam - graças à sua flexibilidade - o desenvolvimento de estratégias distintivas de legitimação por parte dos especial istas, o que permite compreender a heterogeneidade identitária que atravessa esse universo simbólico. Ao mesmo tempo, essa 'flexibilidade' oferece o terreno analítico para discutir as possibilidades de subversão (ou, pelo contrário, de subsunção) com respeito à lógica dominante imposta, no campo da saúde, desde a biomedicina ou medicina científica.
\end{abstract}

Palavras-chave: terapias alternativas; identidades coletivas; Laclau; Buenos A ires. 